\title{
Acute Lower Gastrointestinal Bleeding in Patients Treated With Non-Vitamin K Antagonist Oral Anticoagulants Compared With Warfarin in Clinical Practice: Characteristics and Clinical Outcome
}

\author{
Georgia Diamantopoulou ${ }^{\mathrm{a}}$, Christos Konstantakis ${ }^{\mathrm{b}}$, George Skroubis ${ }^{\mathrm{c}}$, \\ George Theocharis ${ }^{\mathrm{a}}$, Vasilios Theopistos ${ }^{\mathrm{a}}$, Christos Triantos ${ }^{\mathrm{a}}$, \\ Konstantinos Thomopoulos ${ }^{\mathrm{a}, \mathrm{d}}$
}

\begin{abstract}
Background: Acute lower gastrointestinal bleeding (ALGIB) can occur in patients on anticoagulant therapy (either warfarin or nonvitamin $\mathrm{K}$ oral anticoagulants (NOACs)). Use of NOACs has been increasing compared to warfarin in recent years. We analyzed patients with ALGIB on anticoagulation therapy and compared characteristics, management and clinical outcome in patients treated with NOACs versus warfarin.

Methods: All patients with ALGIB on anticoagulation therapy treated in two (affiliated) centers during a 7-year period were evaluated. Characteristics and clinical outcome were compared between patients on warfarin and patients on NOACs.

Results: Out of the 587 patients identified with ALGIB during the study period, $43(7.3 \%)$ were on NOACs and $68(11.6 \%)$ on warfarin. Mean age was $75.9 \pm 9.5$ and $77.1 \pm 7.9$ years respectively. Site of bleeding was located in the small bowel in 2/43 of NOAC patients and $6 / 68$ of warfarin group. Vascular ectasias (8/43 vs. $6 / 68$, $\mathrm{P}=0.010)$ and polyps/neoplasia $(13 / 43$ vs. $6 / 68, \mathrm{P}=0.025)$ were more commonly causes of bleeding in patients on NOACs. While endoscopic hemostasis was more commonly needed in patients on NOACs $(17 / 43$ vs. $14 / 68, P=0.049)$, they required less hospitalization days $(4.5 \pm 3.6$ vs. $6.1 \pm 4.2, \mathrm{P}=0.032)$. Blood transfusions and need for other interventions (embolization and/or surgery) as well as recurrence of bleeding and mortality were not statistically different.
\end{abstract}

\footnotetext{
Manuscript submitted November 8, 2018, accepted November 23, 2018

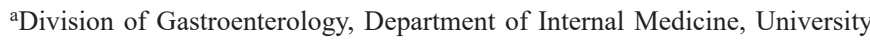
Hospital of Patras, 26504 Rio, Greece

bepartment of Gastroenterology, General Hospital of Patras, 26300 Patras, Greece

'Department of Surgery, University Hospital of Patras, 26504 Rio, Greece ${ }^{\mathrm{d} C o r r e s p o n d i n g ~ A u t h o r: ~ K o n s t a n t i n o s ~ T h o m o p o u l o s, ~ D i v i s i o n ~ o f ~ G a s t r o e n t e r-~}$ ology, Department of Internal Medicine, University Hospital of Patras, 26504 Rio, Greece. Email: kxthomo@hotmail.com
}

doi: https://doi.org/10.14740/gr1115
Conclusions: Although NOAC patients with ALGIB exhibit some differences on certain clinical characteristics when compared to warfarin patients, they share a similar clinical outcome.

Keywords: Lower gastrointestinal bleeding; Anticoagulation; Warfarin; NOACs

\section{Introduction}

Vitamin K antagonists, mainly warfarin, have been successfully used for the treatment and prevention of venous and arterial thromboembolism for many decades. Recent pharmacological research progress has seen the development of several nonvitamin K oral anticoagulants (NOACs). Dabigatran (a direct thrombin inhibitor) rivaroxaban and apixaban (direct factor Xa inhibitors) are among the most commonly prescribed. NOACs when compared to warfarin have been proven to be at least as effective (non-inferior or non-superior) in preventing thromboembolic events. Due to certain limitations of vitamin K antagonists (need for anticoagulant activity monitoring in the form of blood testing, certain drug to drug and food to drug interactions) and similar efficacy in preventing thromboembolic events the popularity of NOACs has been steadily increasing, especially in the elderly [1-3].

Bleeding side-effects (mainly intracranial and gastrointestinal) is a significant complication encountered both in vitamin $\mathrm{K}$ antagonists and NOACs patients [4]. Gastrointestinal bleeding may originate from anywhere between the upper and the lower gastrointestinal tract (small and large bowel). In a recent meta-analysis, patients who were treated with NOACs showed increased risk of GIB compared to those receiving standard care [5]. While management of patients with upper gastrointestinal bleeding is much more straightforward, patients with lower gastrointestinal bleeding present many diagnostic and therapeutic dilemmas. There is scarcity of data on the characteristics, management and clinical outcome of patients with acute lower gastrointestinal bleeding (ALGIB) while on anticoagulation therapy, especially those on NOACs $[6,7]$. These 
patients are usually older with more comorbidities that may influence the management and the clinical outcome. With the exception of dabigatran there are no approved reversal agents for the other NOACs in contrast to warfarin. Furthermore, idarucizumab (Praxbind ${ }^{\circledR}$ : reversal of dabigatran) was not available until recently (approved in November 2015 in our country).

The aim of this study was to analyze characteristics, management and clinical outcome of ALGIB patients treated with NOACs or warfarin and to investigate differences between the two groups regarding clinical characteristics, etiology, use of blood products, need for hemostatic interventions, hospitalization and clinical outcome.

\section{Patients and Methods}

Medical records of all patients with ALGIB on anticoagulation therapy treated in two affiliated hospitals between January 2010 and December 2016 were retrospectively reviewed. We included all adult (above 18 years) patients on anticoagulants who were either admitted for ALGIB or who were hospitalized for any reason and were complicated with ALGIB. Patients presented with: 1) acute hematochezia, from bright red to marrow blood with clots or 2) melena with normal upper endoscopy and absence of blood in the esophagus, stomach and duodenum. Patients with acute hematochezia or melena with documented lesion in the upper gastrointestinal tract and patients with chronic blood loss and/or iron deficiency anemia were likewise excluded from this study.

In all patients, oral coagulant therapy was stopped and fresh frozen plasma was given to patients on warfarin with prolonged international normalized ratio (INR). Endoscopy, according to our current practice, was performed after partial correction of the INR with fresh frozen plasma. Patients at low risk of thromboembolism (atrial fibrillation, remote history of deep venous thrombosis) were taken off anti-coagulants for a short period, and then in accordance to the endoscopic findings, anticoagulation was restarted after a few days. In other patients low molecular heparin was started when risk of rebleeding was low according to the endoscopic findings and heparin therapy was switched to oral anticoagulant therapy after a few days. Blood transfusions were given to achieve a hemoglobin level of $9 \mathrm{mg} / \mathrm{dL}$ in non-cirrhotic or $8 \mathrm{mg} / \mathrm{dL}$ in cirrhotic patients. During the limited time that idarucizumab was available (only available several months before the end of the study period) no patients were treated with this reversal agent.

All patients in both hospitals were treated with an intensive diagnostic and therapeutic protocol. Clinical evaluation, resuscitation and appropriate replacement of blood loss were the main steps in the management of the patients. Endoscopy of large bowel was performed during the first $24 \mathrm{~h}$ of admission after colon preparation in the majority of patients. In cases of hemodynamic instability, emergent computed tomography angiography (CTA) was performed with embolization when appropriate. Patients with melena had gastroscopy for exclusion of upper gastrointestinal bleeding. When small bowel bleeding was suspected, capsule endoscopy was carried.
Table 1. Type of Anticoagulation Therapy of Patients

\begin{tabular}{cll}
\hline & N & \% \\
\hline Warfarin & $68 / 587$ & 11.6 \\
NOACs & $43 / 587$ & 7.3 \\
Dabigatran & 23 & 3.9 \\
Apixaban & 3 & 0.5 \\
Rivaroxaban & 17 & 2.9 \\
\hline
\end{tabular}

NOACs: non vitamin $\mathrm{K}$ oral anticoagulants.

Therapeutic endoscopic interventions included: injection hemostasis with adrenaline diluted at 1:10,000 in saline $0.9 \%$ in patients with active bleeding or stigmata of recent bleeding, the use of endoclips especially in post-polypectomy cases and the application of thermal contact treatment, such as argon plasma coagulation (APC) in patients with vascular ectasias.

Demographic and clinical characteristics of all patients were recorded. Patient's age, gender, clinical presentation of bleeding, type of anticoagulant, laboratory tests on admission (hemoglobin, hematocrit, platelet count, urea, creatinine), in-hospital onset of gastrointestinal bleeding, comorbidities, Charlson comorbidity index (CCI), history of gastrointestinal bleeding, history of gastrointestinal surgery (any kind of operation of the gastrointestinal tract), presence of active bleeding on endoscopy, therapeutic intervention (endoscopic hemostasis, embolization or surgery) and clinical outcome (days of hospitalization, need for blood transfusion, bleeding relapse, death) were all registered.

Diverticula were considered the cause of bleeding when active bleeding or stigmata of recent bleeding were observed or according to clinical presentation and course in the presence of diverticula and absence of other causes of bleeding. Ischemic colitis was diagnosed based on the findings of colonoscopy, pathology and clinical course.

Characteristics and clinical outcome were compared between patients on warfarin and patients on NOACs. Differences between patients on warfarin and NOACs were assessed. Continuous variables were expressed as mean \pm standard deviation and were compared using Student's $t$-test. Categorical variables were expressed as percentages and differences between groups were tested for significance by using the Chisquare test. All analyses were conducted by using statistical software (SPSS v 16.0; IBM Corp, Armonk, NY).

\section{Results}

From a total of 587 patients who were hospitalized in both centers with ALGIB in a period of 7 years, 111 were on anticoagulation therapy. Forty three patients $(7.3 \%)$ were on NOACs, and $68(11.6 \%)$ on warfarin with a mean age of 75.9 \pm 9.5 and $77.1 \pm 7.9$ years, respectively. Among 43 patients on NOACs, 23 (53.4\%) were on dabigatran, 17 (39.5\%) were on rivaroxaban and three $(6.9 \%)$ were on apixaban (Table 1$)$. It seems that the proportion of patients with ALGIB taking NOACs has been increasing over the years in contrast to those 


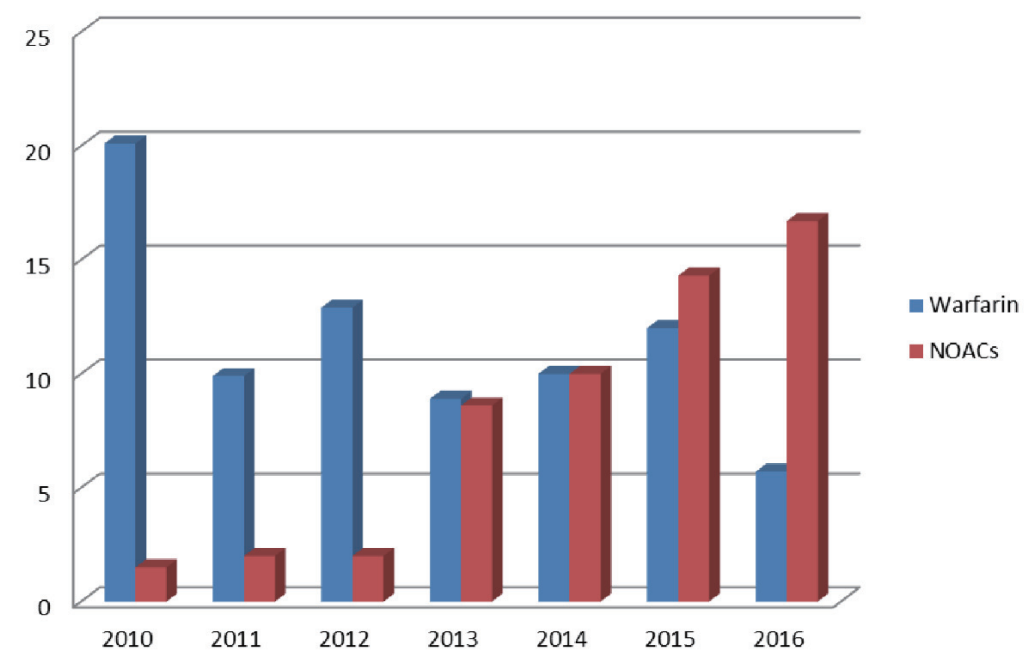

Figure 1. Percent of patients with ALGIB taking warfarin or NOACs per year.

taking warfarin (Fig. 1).

Indications for anticoagulation included atrial fibrillation (AT) in 87 cases, pneumonic embolism (PE) or deep vein thrombosis (DVT) in eight cases and prosthetic valve in 16 cases. All patients had at least one comorbidity and the mean Charlson comorbidity index was not statistically different between the two groups (5.1 vs. 5.4, $\mathrm{P}=0.948$ ) (Table 2).

The laboratory tests on admission like hemoglobin, platelets count, blood urea nitrogen and creatinine were similar between the two groups of patients (Table 2).

The bleeding site was in the small bowel in 2/43 and 6/68 respectively. Causes of bleeding were not different between the two groups with the exceptions of vascular ectasias $(8 / 43$ vs. $6 / 68, \mathrm{P}=0.010)$ and polyps/neoplasia (13/43 vs. $8 / 68, \mathrm{P}=$ 0.025 ) which were more frequent in the NOACs group (Table 2).

While endoscopic hemostasis was more frequently needed in patients on NOACs $(17 / 43$ vs. $14 / 68, \mathrm{P}=0.049)$, they required less hospitalization days $(4.5 \pm 3.6$ vs. $6.1 \pm 4.2, \mathrm{P}=$ 0.032). Needs for blood transfusions and other interventions (embolization and/or surgery) were not different between the two groups. Recurrence of bleeding (4/43 vs. $11 / 68, \mathrm{P}=0.398$ ) was higher in the warfarin group and mortality (3/43 vs. $0 / 68$, $\mathrm{P}=0.056)$ lower, but neither characteristic reached statistical significance (Table 3).

\section{Discussion}

Gastrointestinal bleeding is a well-documented complication of anticoagulation therapy (amounting for up to $50 \%$ of the total of bleeding events in these patients). In our study a substantial proportion of patients with ALGIB bled while on anticoagulation therapy with either warfarin $(11.6 \%)$ or NOACs $(7.3 \%)$. There is controversy as to whether the risk of ALGIB is higher in patients taking NOACs. In previous studies an increased risk of gastrointestinal bleeding was observed especially for dabigatran compared to conventional anticoagulation
$[3,8]$. However, in a recent meta-analysis including 43 randomized trials no difference in the risk of lower gastrointestinal bleeding between NOACs and conventional anticoagulants was found [9]. Older patients and higher dosage may indeed increase the risk of bleeding. It has been shown that low dabigatran dose was not associated with higher risk of bleeding; a higher dose (150 mg, twice a day) was associated with increased gastrointestinal bleeding risk only in those aged $\geq 75$ years when compared with warfarin [10].

In our study we included only patients with major lower gastrointestinal bleeding who needed hospitalization. All patients had at least one comorbidity, but most had a favorable outcome. Regardless, recurrence rates of bleeding, need for transfusion, need for intervention or surgery and mortality were low, and no difference between the two groups. NOACs have a short half-life, and usually cessation of treatment will lead to a rabid restoration of the coagulative function within 12 - $24 \mathrm{~h}$ [11]. Although mortality was lower in patients taking warfarin this difference did not reach a statistically significant level; however the two groups were not prospectively evaluated to reach firm conclusions. Patients with bleeding on NOACs required less hospitalization compared with patients on warfarin, probably because patients on vitamin $\mathrm{K}$ antagonists need more time to adjust bleeding time. Similar findings with the exception of transfusion needs were found in another study where acute bleeding from both upper and lower gastrointestinal tract in patients on NOACs were compared to those on warfarin. In this study need for transfusion was more common in patients on warfarin but this study only included five patients in the NOACs group [12].

Patients on NOACs more commonly bleed from polyps and/or neoplasia compared to patients on warfarin $(18.6 \% \mathrm{vs}$. $8.8 \%$ ). Flack et al reviewing the results from the RE-LY trial found that $8 \%$ of all acute gastrointestinal bleeding events were related to gastrointestinal malignancies ( $80 \%$ colorectal), and bleedings occurred early after introduction of anticoagulative agent and were persisting compared with gastrointestinal bleeding events from other causes [13]. 
Table 2. Clinical and Endoscopic Characteristics of Patients With Acute Lower Gastrointestinal Tract Bleeding

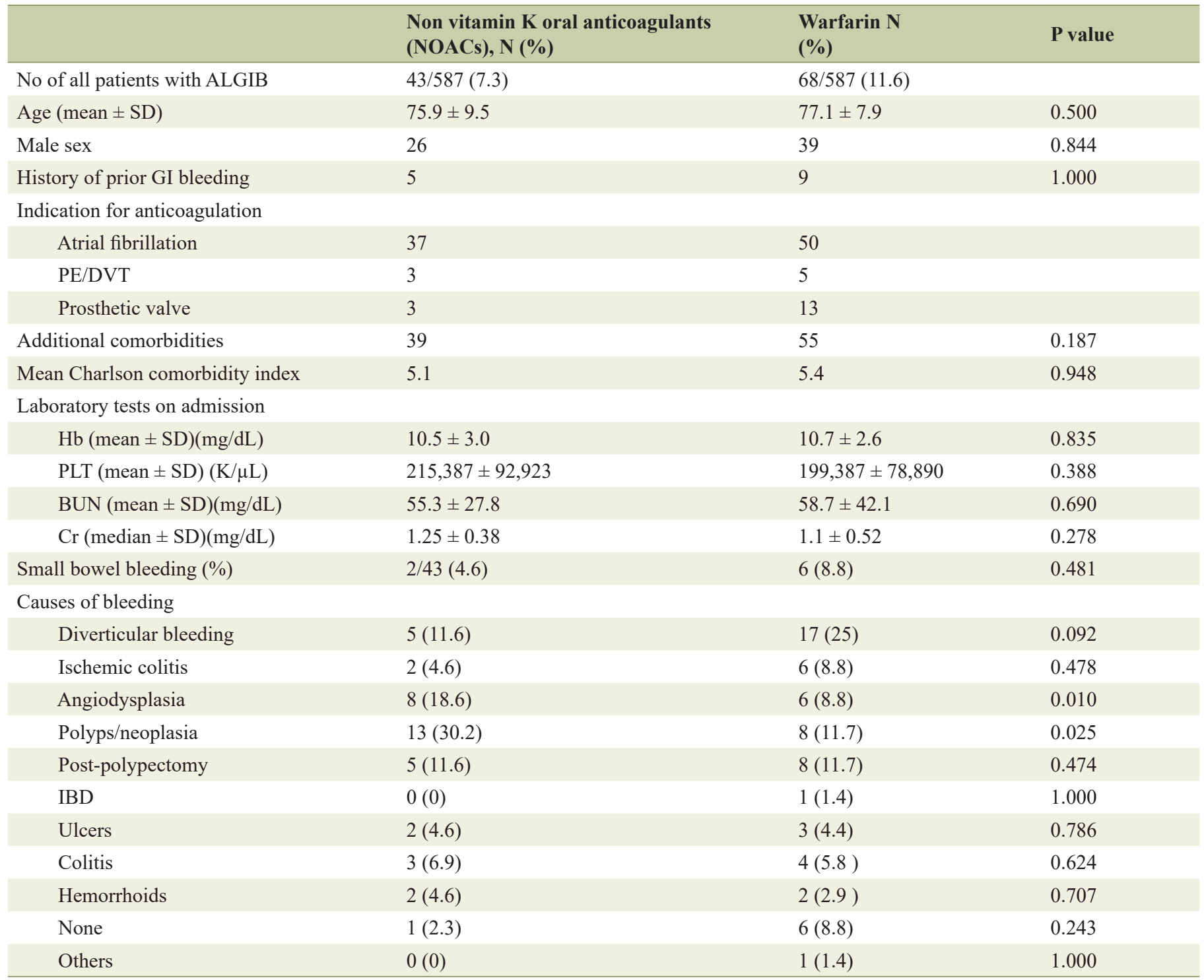

ALGIB: acute lower gastrointestinal bleeding; SD: standard deviation; PE: pneumonic embolism; DVT: deep vein thrombosis; Hb: hemoglobin; PLT: platelets; BUN: blood urea nitrogen; Cr: creatinine; IBD: inflammatory bowel disease.

Although the anticoagulant effect of warfarin is achieved through systematic inhibition of vitamin K-dependent clotting factors, NOACs could also have a more topical effect. NOACs probably show some degree of intraluminal anticoagulant activity as these agents are not completely absorbed across the gastrointestinal tract and may cause intraluminal bleeding from pre-existing vulnerable lesions like polyps. On the other hand warfarin is completely absorbed and has no intraluminal anticoagulation [14]. Moreover, for dabigatran an additional caustic effect has been proposed implicating the tartaric acid that's present in the final formulation of the drug [15]. In a recent retrospective study from Hong Kong patients on proton pump inhibitors (PPI) or histamine 2 receptor antagonists (H2RA) who received dabigatran, had less gastrointestinal bleeding events compared to those not on gastroprotection [16].
Management of patients taking NOACs or warfarin who present with ALGIB involves cessation of the anticoagulant and endoscopic treatment if and when it is required [17]. The higher rate of endoscopic hemostasis in patients on NOACs is probably associated with the higher frequency of polypoid lesions as the cause of bleeding where endoscopic hemostasis of some way is usually applied. Hemodialysis or hemoperfusion is an option for dabigatran in the rare case of life-threatening bleeding or renal failure but not for direct factor Xa inhibitors as these factors are tightly protein-bound in the blood $[14,18]$. During the study period there was no specific reversal agent readily available for NOACs. Idarucizumab (Praxbind ${ }^{\circledR}$, a reversal agent for dabigatran) was not available until recently in our country as it was approved in late 2015 and was commercially available on early 2016. Combined with the facts of its 
Table 3. Clinical Outcome of Patients With ALGI Bleeding Taking Anticoagulants

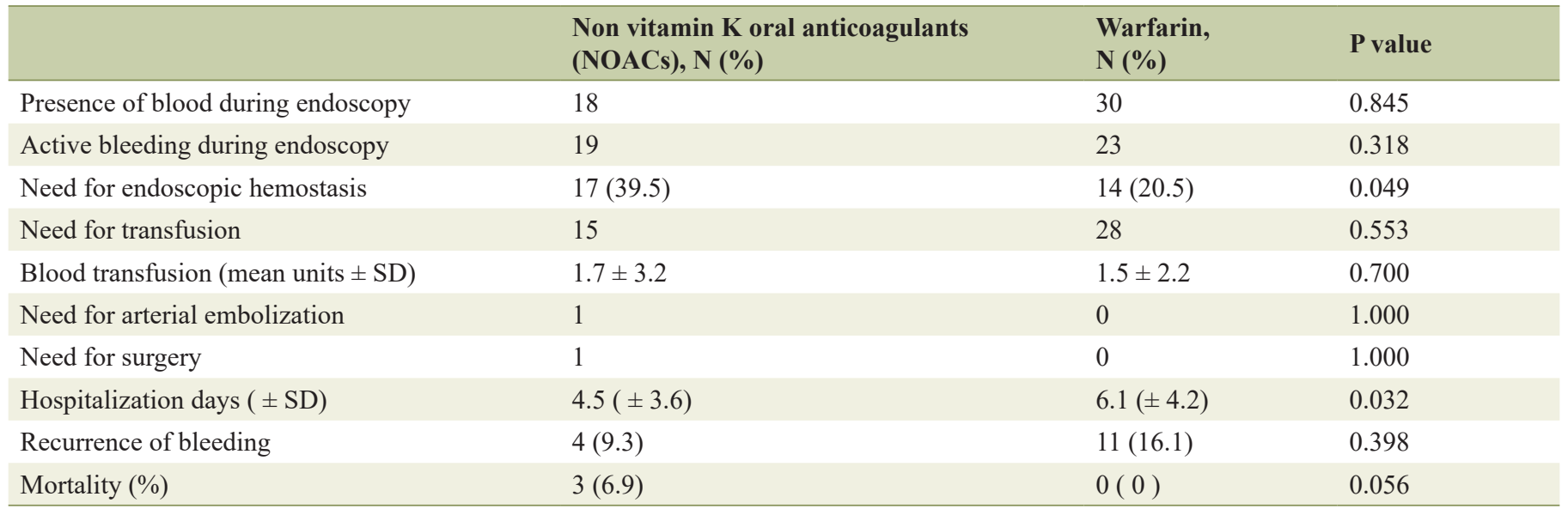

SD: standard deviation.

considerable cost and the indication warnings (labeled as a last resort treatment option), which all made its use rather limited. In fact, during the study period no patients were treated with idarucizumab.

Our study has a number of limitations. It was retrospective and some minor cases of ALGIB might not have been included. Although the study included patients from both a tertiary center and a smaller affiliated hospital, all the facilities and resources of the referral unit were available when needed; and finally, because of the rarity of this complication, the number of patients was relatively small. Prospective large scale studies are required.

In conclusion, although ALGIB in patients on NOACs presents some differences, it has a similar clinical outcome compared to patients with ALGIB on warfarin. Although reversal agents to NOACs are not widely available in our clinical practice, the problem of ALGIB is not major provided that correct dosing of the agent and appropriate selection of patients especially those with renal impairment are followed.

\section{References}

1. Connolly SJ, Ezekowitz MD, Yusuf S, Eikelboom J, Oldgren J, Parekh A, Pogue J, et al. Dabigatran versus warfarin in patients with atrial fibrillation. N Engl J Med. 2009;361(12):1139-1151.

2. Granger CB, Alexander JH, McMurray JJ, Lopes RD, Hylek EM, Hanna M, Al-Khalidi HR, et al. Apixaban versus warfarin in patients with atrial fibrillation. N Engl J Med. 2011;365(11):981-992.

3. Patel MR, Mahaffey KW, Garg J, Pan G, Singer DE, Hacke W, Breithardt G, et al. Rivaroxaban versus warfarin in nonvalvular atrial fibrillation. N Engl J Med. 2011;365(10):883-891.

4. Diener HC, Aisenberg J, Ansell J, Atar D, Breithardt G, Eikelboom J, Ezekowitz MD, et al. Choosing a particular oral anticoagulant and dose for stroke prevention in individual patients with non-valvular atrial fibrillation: part 2 .
Eur Heart J. 2017;38(12):860-868.

5. Steinberg BA, Simon DN, Thomas L, Ansell J, Fonarow GC, Gersh BJ, Kowey PR, et al. Management of major bleeding in patients with atrial fibrillation treated with non-vitamin $\mathrm{K}$ antagonist oral anticoagulants compared with warfarin in clinical practice (from Phase II of the Outcomes Registry for Better Informed Treatment of Atrial Fibrillation [ORBIT-AF II]). Am J Cardiol. 2017;119(10):1590-1595.

6. Holster IL, Valkhoff VE, Kuipers EJ, Tjwa E. New oral anticoagulants increase risk for gastrointestinal bleeding: a systematic review and meta-analysis. Gastroenterology. 2013;145(1):105-112 e115.

7. Roskell NS, Samuel M, Noack H, Monz BU. Major bleeding in patients with atrial fibrillation receiving vitamin $\mathrm{K}$ antagonists: a systematic review of randomized and observational studies. Europace. 2013;15(6):787-797.

8. He Y, Wong IC, Li X, Anand S, Leung WK, Siu CW, Chan EW. The association between non-vitamin K antagonist oral anticoagulants and gastrointestinal bleeding: a meta-analysis of observational studies. Br J Clin Pharmacol. 2016;82(1):285-300.

9. Miller CS, Dorreen A, Martel M, Huynh T, Barkun AN. Risk of Gastrointestinal Bleeding in Patients Taking NonVitamin K Antagonist Oral Anticoagulants: A Systematic Review and Meta-analysis. Clin Gastroenterol Hepatol. 2017;15(11):1674-1683 e11673.

10. Eikelboom JW, Wallentin L, Connolly SJ, Ezekowitz M, Healey JS, Oldgren J, Yang S, et al. Risk of bleeding with 2 doses of dabigatran compared with warfarin in older and younger patients with atrial fibrillation: an analysis of the randomized evaluation of long-term anticoagulant therapy (RE-LY) trial. Circulation. 2011;123(21):23632372.

11. Desai J, Granger CB, Weitz JI, Aisenberg J. Novel oral anticoagulants in gastroenterology practice. Gastrointest Endosc. 2013;78(2):227-239.

12. Cangemi DJ, Krill T, Weideman R, Cipher DJ, Spechler SJ, Feagins LA. A comparison of the rate of gastrointes- 
tinal bleeding in patients taking Non-Vitamin K antagonist oral anticoagulants or warfarin. Am J Gastroenterol. 2017;112(5):734-739.

13. Flack KF, Desai J, Kolb JM, Chatterjee P, Wallentin LC, Ezekowitz M, Yusuf S, et al. Major gastrointestinal bleeding often is caused by occult malignancy in patients receiving warfarin or dabigatran to prevent stroke and systemic embolism from atrial fibrillation. Clin Gastroenterol Hepatol. 2017;15(5):682-690.

14. Desai J, Kolb JM, Weitz JI, Aisenberg J. Gastrointestinal bleeding with the new oral anticoagulants - defining the issues and the management strategies. Thromb Haemost. 2013;110(2):205-212.

15. Singh S, Savage L, Klein M, Thomas C. Severe necrotic oesophageal and gastric ulceration associated with dabi- gatran. BMJ Case Rep. 2013;2013.

16. Chan EW, Lau WC, Leung WK, Mok MT, He Y, Tong TS, Wong IC. Prevention of dabigatran-related gastrointestinal bleeding with gastroprotective agents: a population-based study. Gastroenterology. 2015;149(3):586-595 e583.

17. Weitz JI, Pollack CV, Jr. Practical management of bleeding in patients receiving non-vitamin $\mathrm{K}$ antagonist oral anticoagulants. Thromb Haemost. 2015;114(6):11131126.

18. Khadzhynov D, Wagner F, Formella S, Wiegert E, Moschetti V, Slowinski T, Neumayer HH, et al. Effective elimination of dabigatran by haemodialysis. A phase I single-centre study in patients with end-stage renal disease. Thromb Haemost. 2013;109(4):596-605. 